\title{
Reflets
}

Revue d'intervention sociale et communautaire

\section{Regards humanistes sur le pluralisme social et culturel dans l'espace clinique : les points de vue d'un pédiatre et d'un anthropologue}

\section{Entrevue avec Fernando Alvarez et Gilles Bibeau}

\section{Marguerite Soulière}

Volume 17, numéro 2, automne 2011

Représentations, pratiques et expérience dans le champ de santé :

tendre des ponts entre des disciplines

URI : https://id.erudit.org/iderudit/1012128ar

DOI : https://doi.org/10.7202/1012128ar

Aller au sommaire du numéro

Éditeur(s)

Reflets, Revue d'intervention sociale et communautaire

ISSN

1203-4576 (imprimé)

1712-8498 (numérique)

Découvrir la revue

Citer ce document

Soulière, M. (2011). Regards humanistes sur le pluralisme social et culturel

dans l'espace clinique : les points de vue d'un pédiatre et d'un anthropologue :

entrevue avec Fernando Alvarez et Gilles Bibeau. Reflets, 17(2), 30-48.

https://doi.org/10.7202/1012128ar 


\section{Regards humanistes sur le pluralisme social et culturel dans l'espace clinique : les points de vue d'un pédiatre et d'un anthropologue}

\section{Entrevue avec Fernando Alvarez et Gilles Bibeau}

Fernando Alvarez, médecin et directeur du Service de gastroentérologie, hépatologie et nutrition, Centre hospitalier universitaire Sainte-Justine et cofondateur de l'Unité de pédiatrie interculturelle, et

Gilles Bibeau, professeur au département d'anthropologie de l'Université de Montréal, spécialiste dans le domaine des études africaines et en anthropologie de la santé, et cofondateur de l'Unité de pédiatrie interculturelle.

L'entrevue a été réalisée par Marguerite Soulière.

\section{Préambule}

En 2003, le Département de pédiatrie de l'Université de Montréal du Centre hospitalier universitaire Sainte-Justine (CHU SainteJustine), en conformité avec sa mission de base, s'est donné pour mandat de développer une unité de recherche et de formation axée sur le pluralisme social et culturel. De là est née l'Unité de pédiatrie interculturelle (UPI). La diversité croissante de la population desservie à l'hôpital appelle une adaptation des pratiques cliniques aux patients et à leurs familles. 
M. Soulière: Qu'est-ce qui vous a amenés à vous intéresser, l'un comme anthropologue, l'autre comme médecin, à la question du pluralisme dans l'espace clinique à l'hôpital?

G. Bibeau : Pour ma part, l'histoire remonte bien avant ma rencontre avec Fernando Alvarez. Avant cette rencontre,j'avais une vie! [rires] Je dirais qu'il y a trois racines à cette coopération que nous menons, Fernando et moi, depuis quelques années. Dès notre première discussion, nous sommes tombés d'accord sur la nécessité de transformer l'hôpital de l'intérieur.Voici ces trois racines.

Pour évoquer la première racine, il faut remonter trente-cinq ans en arrière, à une époque où j'ai réalisé au Zaïre un film qui s'intitulait Un hôpital en forme de village. À l'époque, je travaillais à l'évaluation de l'efficacité des thérapies traditionnelles, qu'il s'agisse d'herboristerie, ou de thérapies rituelles, spirituelles et médicamenteuses, dans le cadre de l'Institut national de recherche scientifique du Zaïre. Dans ce contexte, j'ai mené un travail ethnographique sur un hôpital situé dans un milieu villageois et tenu par des guérisseurs; on y trouvait une quarantaine de cases dans lesquelles des malades habitaient avec leurs familles. Ce lieu était organisé comme une véritable entité économique avec des champs et des forêts d'où des chasseurs pouvaient ramener de la viande pour les malades. Le couple de guérisseurs à la tête de l'hôpital avait réussi à développer entre les malades une vie sociale riche : tous les matins, une thérapie de groupe réunissait ces derniers autour de l'autel des ancêtres qui était planté au centre du village-hôpital.Les différentes thérapies combinaient des éléments chimiques et des éléments plus spirituels, psychologiques et sociaux. L'hôpital pouvait donc être pensé comme une sorte de communauté, comme une unité en quelque sorte. Ça, c'est une histoire fort ancienne qui est restée significative pour moi.

La deuxième racine est venue plus tard, quand je me suis intéressé aux systèmes médicaux comparés et aux grandes traditions thérapeutiques du monde, à savoir, les médecines chinoise, ayurvédique, amérindienne et africaine, avec chacune sa façon de voir le corps, de se le représenter, d'expliquer l'origine des maladies et d'en interpréter les symptômes. Et tous ces systèmes, il fallait 
les penser dans leur articulation au système médical occidental (allopathique). Pendant de nombreuses années,j'ai été responsable d'un cours d'introduction aux différentes visions et modes de compréhension de la maladie humaine qui ont été inventés par les différentes sociétés à travers le monde.

La troisième racine est plus contemporaine. Il s'agit de la construction plurielle de notre société. Il ne faut pas oublier que l'hôpital existe toujours dans un univers social donné et qu'il s'organise selon les valeurs dominantes de la société où il se situe. Nous vivons dans des sociétés occidentales dites "postmodernes" qui sont en pleine transformation sur le plan du pluralisme des formes de vie et des valeurs. Nous ne savons pas très bien ce que deviendront nos sociétés, mais nous savons au moins qu'on y trouve une double tension. D'une part, il y a cette tension qui est imposée par la nécessité de maintenir une histoire collective qui permet de créer solidarité et unité entre les citoyens; d'autre part, il y a la présence de valeurs différenciées, ce qui provoque une très forte différenciation. C'est au confluent de cette tension qu'il faut situer l'apparition de l'« interculturalisme " qui a pris différents visages. Voilà la trajectoire qui m'a conduit à l'UPI.

F.Alvarez: Comme vous le savez, nous sommes la conséquence de notre histoire personnelle, la conséquence de notre éducation. Ma formation en pédiatrie a eu lieu dans un hôpital en Argentine où commettre une petite erreur d'orientation diagnostic faisait partie de la formation. Si tu te trompais, quelqu'un allait te corriger, jamais de façon hautaine ou agressive, car tout le monde reconnaissait que tu étais en formation. Mais même en formation, une faute dans le rapport humain avec un enfant ou sa famille n'était jamais pardonnée. C'était quelque chose de considéré comme étant très grave. La personne qui a été pour moi un grand formateur, mon mentor, était quelqu'un qui priorisait l'humain. J'ai donc été formé dans un hôpital qui avait une philosophie profondément humaniste. D'ailleurs, cet hôpital a été l'un des premiers hôpitaux au monde qui, dans les années cinquante, ont permis aux mères de rester auprès de leur enfant hospitalisé. 
Lorsque je suis allé travailler en France, ç'a été pour moi un choc culturel. Là-bas, il n'était pas question que les parents restent auprès de leur enfant hospitalisé. C'était une façon de faire très fréquente. Les Français sur ce point étaient quarante ans, cinquante ans en retard vis-à-vis de ce que j'avais vécu et appris durant ma formation.

M. Soulière: Et plus concrètement, comment l'anthropologie et la médecine se sont-elles rencontrées au CHU Sainte-Justine? Ou comment en êtes-vous arrivés à travailler ensemble et à mettre sur pied l'Unité de pédiatrie interculturelle (UPI)?

F. Alvarez : Ici, au CHU Sainte-Justine, notre approche est peut-être un peu plus humaine, même si elle ne l'est pas suffisamment à mon goût. En Amérique du Nord, le rapport Fechner rédigé au début du $\mathrm{XX}^{\mathrm{e}}$ siècle a clairement montré que la formation médicale était centrée sur les sciences biologiques au détriment de la formation humaine. Même si l'objectif de notre travail et la raison d'être de notre profession sont la prise en charge de l'être humain souffrant, ces souffrances ne proviennent pas uniquement d'une anomalie biologique. Considérant ma pratique, je me suis dit que je n'avais pas dans ma formation les éléments qui me permettaient une prise en charge globale de l'être humain. Aussi, pour commencer à influencer mes collègues, je sentais que je n'avais pas les «armes " ou les concepts. Je n'avais pas lu Foucault [rires], je n'avais pas les armes pour aborder ces situations complexes. Je n'ai pas eu à convaincre Gilles Bibeau. Je pense que lorsqu'on s'est rencontrés, il y a eu rapidement une concordance d'objectifs. Gilles peut expliquer.

G. Bibeau : Quand Fernando est venu parler de ce qu'il se faisait au Département de pédiatrie générale, là où se trouvent de nombreuses cliniques de spécialités au sein de l'hôpital - la transplantation dont il assure la direction en est une, mais il y en a encore cinquante autres -, cela m'est apparu extrêmement intéressant. En même temps, j'ai compris qu'il y avait un formidable défi à relever et cela m'a intéressé. 
Il y avait une grande équipe autour de Fernando, notamment des cliniciens qui étaient déjà là et qui souhaitaient des changements; il fallait du côté anthropologique trouver des collègues prêts à collaborer avec les cliniciens. Les premiers à s'impliquer furent des étudiants de maîtrise et de doctorat. D'emblée, trois fonctions complémentaires se sont dégagées, toutes également importantes, qui servirent de leviers pour enclencher un processus de transformation institutionnelle. À partir des questions que les cliniciens se posaient dans les cliniques de spécialités, on a essayé de mettre sur pied des projets en faisant porter des mémoires et des thèses sur ces sujets et éventuellement en développant des projets de recherche qui furent financés par divers organismes subventionnaires. La recherche est ainsi devenue un élément absolument essentiel de l'Unité de pédiatrie interculturelle qui s'est déployée dans une double direction : à l'adaptation des pratiques cliniques et de recherche, nous avons ajouté la question de la formation. Ce dernier volet fut plus difficile à mettre en œuvre. Mais on a travaillé dès le début sur des vignettes cliniques qui servirent à former les résidents; on a continué au fil des années à former les résidents en pédiatrie sans que l'on puisse hélas! ouvrir la formation aux autres spécialités au sein de l'hôpital. Je pense qu'il existait une demande de leur part, mais hélas, on n'avait pas le personnel pour y répondre.

Le volet "formation " est resté inachevé, même si nous étions convaincus qu'il fallait amener les cliniciens à mener un travail d'autoréflexion à partir de l'analyse de cas, dans un retour sur des situations cliniques soumises à l'analyse dans un cadre leur permettant d'identifier la dimension culturelle posée, par exemple, la question de la structure familiale, la dimension économique liée à la pauvreté, etc. Il nous est apparu important de pouvoir amener les cliniciens à distinguer entre tous ces domaines et à les penser dans leurs interactions. La première réponse du clinicien qui est aujourd'hui confronté à une pathologie prend souvent une forme technique, sans doute parce qu'il est formé à travers le recours à l'anamnèse et aux tests biologiques à penser en termes techniques. Toute sa pratique est en effet paramétrée; il connaît les normes qui existent sur tous les plans, les normes de croissance, de 
fonctionnalité pour chacun des systèmes du corps humain. L'idée était d'amener les cliniciens à s'ouvrir à autre chose. Ce qui ne fut pas facile.

\section{Soulière: De quelle façon le pluralisme touche-t-il l'espace de l'hôpital?}

G. Bibeau : Là où la construction d'une société plurielle prend davantage forme, c'est dans les grandes institutions publiques que se donne la société. Il y a d'abord l'école où nous assistons à l'implantation du cours Éthique et culture religieuse dans lequel les enseignantes et enseignants fournissent une introduction à toutes les grandes religions du monde; par le biais de ce cours, les élèves intègrent la tradition religieuse qui est celle de la culture dite d'accueil et, en même temps, ils s'ouvrent aux traditions religieuses d'autres groupes présents dans notre société. Je crois que les jeunes apprennent ainsi à connaître quelque quatorze grandes traditions spirituelles du monde. L'école en est ainsi venue à se transformer en profondeur tout en continuant bien sûr à véhiculer les valeurs dominantes de la société d'accueil, c'est-à-dire, en apprenant aux jeunes à être les premiers, à être compétitifs, à se battre et à devenir des individus autonomes; mais en leur disant aussi qu'il faut de la solidarité, qu'il leur faut créer du lien social, qu'il y aura toujours des plus forts et des plus faibles et que même s'ils doivent être les meilleurs, ils doivent être capables de s'occuper des autres. Ce sont là des exemples de doubles messages envoyés aux élèves. L'école enseigne aussi le pluralisme des valeurs.

Il n'y a pas que les écoles qui se débattent avec cette transformation de la société; les hôpitaux doivent aussi faire face à ce même pluralisme ethnique et culturel. Toutefois, les hôpitaux n'ont pas encore été capables de se transformer véritablement en institutions pluralistes. Je parle ici d'un vrai pluralisme et non pas de la simple présence de médecins venus d'autres pays du monde et pratiquant la médecine occidentale :le pluralisme hospitalier dont je parle vise à rendre les hôpitaux aptes à prendre en charge une population qui appartient à différents mondes, qui possède différentes façons de construire la famille et de vivre les rapports entre les parents et les enfants et qui pense la souffrance à partir de différentes représentations de la maladie. Tout ça doit se faire dans un univers 
de plus en plus technique et avec un appareillage extrêmement puissant dans les modes de prises en charge des patients.

C'est dans ce contexte technologique que la médecine doit se faire humaine, c'est-à-dire, ouverte à ce qui fait de nous des êtres humains. Être capable de prendre en compte l'humain sans rejeter les avancées technologiques, sans jouer l'un contre les autres.Voilà le grand défi de l'hôpital d'aujourd'hui! Il doit en effet être ouvert sur la société dans la mesure où les patients ne vivent pas seulement des situations traumatiques à la suite d'accidents, de traumatismes ou d'épisodes aigus; les cliniciens sont souvent appelés à prendre en charge de nombreux malades sur le long terme. Donc, il fallait être capable de penser l'espace hospitalier dans son articulation sur l'espace domestique, dans un va-et-vient entre hôpital et maison. Cela pose toute une série de nouvelles questions.

M. Soulière: Qu'est-ce que les dix ans de l'UPI ont apporté à l'hôpital SainteJustine?

F. Alavarez : Je pense que les retombées les plus importantes et les plus gratifiantes — je reviens à ce que disait Gilles — c'est l'influence qu'on a pu avoir sur les plus jeunes, c'est-à-dire les résidents, peut-être à cause de nos interventions, des symposiums que nous organisons chaque année. Dans un de ces symposiums, pour la première fois, deux philosophes ont mis les pieds à Sainte-Justine pour nous donner leur point de vue; je crois que ce n'était jamais arrivé auparavant. C'est avec les plus jeunes, peut être parce qu'ils sont plus ouverts, plus sensibles, que je pense qu'il faut continuer à travailler. La seule frustration quant aux retombées de notre travail est le fait que nous n'avons pas réussi à communiquer avec les autres catégories professionnelles. Nous avons peu de contact avec les infirmières et je crois que c'est une chose que nous devrions améliorer et que nous avons l'obligation morale de le faire. Lors des réunions de l'équipe de transplantation hépatique que je dirige, les infirmières sont très attentives à ce qu'on dit et elles ne manquent jamais. Il y en a toujours au moins trois de présentes et elles font entièrement partie de l'équipe : l'infirmière des soins intensifs, l'infirmière de l'étage et l'infirmière coordonnatrice pour la greffe. Chaque fois qu'on se réunit, elles sont parmi les plus passionnées. Elles savent 
aussi que dans notre équipe, on leur donne la parole. Je crois que c'est sur le rapport entre l'UPI et l'ensemble des infirmières de l'hôpital que nous devons travailler dans les années qui viennent. Et Gilles aussi en est convaincu. Nous l'avons essayé, sans beaucoup de succès; mais je pense que nous allons finir par sensibiliser la direction à nous fournir les moyens de le faire. Vers la fin de l'année, nous aurons l'occasion de reprendre activement notre approche. À ce moment-là, les changements qu'on va produire à Sainte-Justine seront beaucoup plus importants. Surtout qu'il y a un renouvellement des infirmières, les équipes sont beaucoup plus jeunes et elles ont l'oreille attentive. Je le vois parce que lorsque je leur parle dans les mêmes termes que ceux nous utilisons en ce moment, elles sont très attentives.

M. Soulière : Ce carrefour entre l'espace public de l'hôpital et l'espace privé de la maison pose quel genre de défi?

G. Bibeau : C'est quoi l'espace de la maison?Voilà la première question à nous poser. Est-ce que la famille est toujours composée de papa, de maman et des enfants? Elle est devenue beaucoup plus complexe que cela et pas seulement chez les populations immigrantes, mais dans l'ensemble de la société où nous trouvons des ménages reconstitués, avec de nombreuses figures paternelles et maternelles qui sont là autour des enfants et qui sont — on est obligé d'en tenir compte - de niveaux économiques variables. Donc forcément, quand on parle de la maison, on parle aussi de hiérarchie sociale; on parle d'une classe pauvre, de gens qui ont des difficultés, sans être analphabètes, et qui possèdent à peine, si on peut dire, la capacité de dire leur mal, de dire leur souffrance. Pour moi, cette articulation de l'hôpital sur l'espace domestique est quelque chose d'absolument essentiel à prendre en compte.

F. Alvarez: D'ailleurs, on a eu beaucoup de discussions par le passé sur ce qu'on appelle "Unité de pédiatrie interculturelle " alors qu'elle devait s'appeler "Unité de pédiatrie interculturelle et sociale». Les différences sociales dans notre société sont des dimensions présentes dans la rencontre et dans les rapports entre le personnel soignant et les familles. Ces variables sociales influencent parfois beaucoup plus les rapports entre le médecin et les familles que 
les dimensions dites interculturelles. Moi, je suis un immigré ici, même si je me sens néo-québécois, si on me demande pourquoi je n'ai pas eu de difficulté d'intégration, je réponds que c'est simplement parce que le dialogue entre universitaires est beaucoup plus facile que le dialogue avec quelqu'un qui n'a pas fini son école primaire. En ce sens que c'est plus facile lorsqu'on partage le même niveau d'instruction et les mêmes caractéristiques socioéconomiques.

M. Soulière: Revenons à la question du pluralisme et plus spécifiquement à celle de l'altérité...

F.Alvarez: Lorsque nous parlons de pluralisme et d'altérité, les défis sont énormes. On s'en est rendu compte lorsque nous avons participé à la commission Bouchard-Taylor avec d'autres collègues. Les discours laissaient transparaitre des préjugés sans aller plus loin dans l'analyse. C'était incroyable! Nous, nous ne parlions pas parce que nous étions stupéfaits et à un moment donné, M. Bouchard a demandé : "Mais vous, qu'est-ce que vous pensez? »

Pour nous, les accommodements raisonnables n'existent pas. Notre rôle est d'essayer de comprendre et d'aider les familles.Alors pour nous, ce n'est pas un " accommodement » qui est fait, cela fait partie de notre métier. C'est une vision complètement différente de l'affaire. L'effort que nous faisons avec nos collègues à l'UPI, c'est de sensibiliser le personnel médical à ne pas culturaliser la différence.

G. Bibeau : J'aimerais réagir doublement sur ce que vient de dire Fernando : premièrement, sur l'altérité et la différence, deuxièmement, sur la nécessité d'humaniser la médecine, et particulièrement l'espace clinique. Du côté de l'altérité, je dirais que le point de vue que nous avons adopté à l'UPI en est un extrêmement pragmatique, guidé par un principe fondamental, à savoir qu'on n'a pas le droit de renvoyer d'emblée la personne à sa culture d'origine. Nous ne savons pas en effet où se situent les familles et les personnes immigrantes dans leur processus de reconstruction identitaire; il pourrait très bien arriver qu'une famille immigrante d'Afrique centrale soit plus occidentalisée que je le sois moi-même et qu'elle 
soit même complètement détachée, et depuis longtemps, de sa culture d'origine. Il faut donc être extrêmement prudent, car il n'y a pas d'indicateurs objectifs et visibles qui nous permettent de situer d'emblée une personne ou une famille par rapport à ce qu'on peut appeler la culture d'origine et la culture d'accueil. À cette dimension culturelle, il faut ajouter les éléments socioéconomiques, c'est-à-dire, la prise en compte des conditions concrètes et quotidiennes d'existence des familles. De ce point de vue là, nous sommes aussi dans l'altérité en ce sens qu'il existe des inégalités au sein d'une même culture Ce sont là les principes «de base ».

F. Alvarez: L'une des choses que j'ai découvertes en travaillant avec l'UPI, c'est que j'ai un grand avantage : celui d'être un étranger. J'ai même souvent un meilleur rapport avec les familles - ce que je ne devrais pas le dire, car ce n'est pas très modeste! J'ai un meilleur rapport que mes collègues, notamment, avec tous les Amérindiens qui nous consultent. Hier, il y avait la maman d'un enfant amérindien hospitalisé et ma collègue lui a expliqué pendant deux jours de suite ce que nous allions faire. Et la maman a dit : "J'aimerais voir le $\mathrm{D}^{\mathrm{r}}$ Alvarez. " J'ai dit : « Madame, ma collègue vous a tout expliqué. " Alors, elle a dit : "Oui, oui! Mais je n'ai rien compris. Expliquez-moi.» Et je lui ai expliqué; son mari est arrivé et je lui ai réexpliqué; et elle a appelé la grand-mère de l'enfant à la réserve pour que je lui explique aussi au téléphone.

C'est peut-être à travers le lien de confiance, non seulement à travers la parole, mais aussi à travers les gestes, à travers le regard, à travers tellement de choses qui composent la communication. Peut-être qu'ils se sentent plus en confiance ou peut-être que mon accent les met en confiance, je ne sais pas. Mais c'est quelque chose qui m'est évident. Peut-être aussi que c'est parce qu'on a prêté attention, on a d'abord écouté, on a utilisé les mots qui les touchent, les mots qui nous permettent d'établir le dialogue.

M. Soulière: Finalement, vous êtes loin d'une vision folklorique de la culture?

G. Bibeau : Cela nous a conduits à un certain conflit par rapport aux perspectives pédagogiques mises de l'avant à l'hôpital, lesquelles 
se fondaient sur l'idée qu'on peut dresser des catégorisations a priori et qu'on peut faire des espèces de tableaux représentant les caractéristiques et les valeurs des différents groupes ethniques. Comme si le Cambodgien avait un profil différent du Haïtien et différent de telle ou de telle autre personne. À l'UPI, on s'est d'emblée opposé à cette perspective-là, en disant que ça ne marche pas comme ça. Mais, je dois dire qu'au fil des années, ça s'est un peu transformé. Encore récemment, on a assisté à un retour de ce processus pédagogique un peu simplificateur, pour ne pas dire véritablement caricatural.

F. Alvarez: À propos de ce que tu dis, après les dix années de l'UPI, j'ai l'impression que nous avons tous des différences culturelles, on ne peut pas les nier, comme le dit Gilles. Déjà du fait qu'on décide de migrer, il y a des choses qui changent, il y a certaines choses qu'on laisse derrière; il faut faire attention, car si l'on caricature les différences culturelles, on risque de laisser les gens en marge de notre société en ne leur permettant pas de s'y incorporer. En même temps, j'ai appris depuis les dix dernières années qu'on réagit selon notre culture aux événements marquants de la vie, qu'ils soient individuels ou sociaux. Mais, il ne faut pas caricaturer ou créer des stéréotypes culturels. Prenez par exemple deux voisines, l'une fait partie de ce groupe de femmes venues de certains villages du Congo, dont la plupart ont été violées ou maltraitées, l'autre vient du village voisin qui n'a pas été affecté par ce conflit, même si elles font partie de la même culture. L'approche humaniste, c'est tenir compte de ces différents vécus au-delà des cultures d'appartenance.

G. Bibeau : Absolument! C'est essentiel. Il y a aussi une chose qui est toujours très bouleversante. Par exemple, quand on parle plusieurs langues, les travaux montrent qu'on désapprend les langues dans le sens inverse où on les a apprises. Ça veut dire quoi, ça? Ça veut dire qu'on meurt en parlant sa langue maternelle et que même si vous avez parlé toute votre vie une autre langue que celle que votre mère vous a apprise, et bien, lorsque dans un coma ou dans un état intermédiaire, les derniers mots que vous direz, vous les direz dans votre langue maternelle. Parce que c'est celle-là qui 
s'est d'abord sédimentée dans votre tête. À partir de cela, je tire une conclusion très importante : lorsque nous sommes menacés dans notre vie, mais très menacés, proches de la mort, même si nous nous sommes détachés des valeurs dans lesquelles nous avons grandi, elles réapparaissent. Elles réapparaissent sans pour autant que cela veuille dire qu'elles vont totalement éliminer les autres. Les menaces extrêmes à la vie font réapparaître chez beaucoup de familles des comportements et des conduites que l'on croyait avoir été effacés par la modernité, par la laïcité, par les valeurs néolibérales.

M. Soulière : Au-delà de la sensibilité culturelle, qu'est-ce qui est indispensable à de bonnes pratiques cliniques?

G. Bibeau : La guérison doit prendre en compte de nombreux éléments extérieurs au corps lui-même. Ces éléments interviennent de manière extrêmement importante dans la remobilisation des systèmes immunitaires, des défenses et du processus complexe de la guérison que nous connaissons encore très mal. Aujourd'hui par exemple, on est en train de réintroduire des déterminants sociaux et économiques dans l'étiologie des problèmes de santé; on est en train de prendre en considération l'impact des dispositions psychologiques sur les mobilisations de défense du corps; on est de plus en plus sensibles aux conditions environnementales, etc. Or, la clinique doit appréhender la personne malade en délimitant une frontière et dire à un moment donné que le clinicien ne peut plus intervenir là-dessus. Je peux dire qu'en tant que clinicien, mon intervention s'arrête ici et que d'autres doivent prendre le relais. Je crois qu'une médecine humaine et une bonne pratique clinique se doivent de prendre en compte la personne et le monde global dans lequel elle vit. Il y a des cliniciens qui font un gros effort pour tendre vers cette approche humaniste, alors que d'autres sont uniquement techniciens et ne peuvent pas entrer véritablement en relation avec l'autre et lui faire sentir que c'est le cœur qui parle. Ça, c'est un des très grands problèmes dans la pratique de la médecine. Et c'est pour ça que certains patients vont dire : "Oui, ce médecin, je le comprends. Je sens qu'il a un cœur. Et il a la technique aussi. " J'ai longtemps pensé que les 
jeunes cliniciens seraient des supertechniciens; et quand on les écoute après les séances, je dois dire que je suis fasciné par leur humanité. Il me faudrait plus d'observations pour dire ce que je vais dire, mais je vais avancer quelque chose, c'est comme si leur humanité, ils devaient la mettre de côté pour être de bons cliniciens et pour bien répondre aux critères de leur textbook de bonnes pratiques

F. Alvarez: Hier, nous avons eu un club de lecture où une résidente a présenté un article qui présentait un traitement très récent pour une molécule qui produit une inflammation de l'intestin. Il s'agissait d'une étude scientifique multicentrique, comme on le fait souvent maintenant, coûtant des millions de dollars et incluant l'Amérique du Nord, l'Europe et l'Europe de l'Est. Les niveaux de rémission étaient au Canada et en Europe de l'Est à peu près $40 \%$ égal à celui du placebo. $40 \%$ d'effet placebo, c'est fort. Dans le reste de l'Europe, il y avait une petite différence parce que le placebo était inférieur à $30 \%$. La question des auteurs était : qu'est-ce qui explique qu'au Canada et en Europe de l'Est, l'effet placebo soit de $40 \%$ et que dans le reste de l'Europe que de $30 \%$ ? Personne ne s'est posé la question sur le fait qu'il y ait autre chose qui influence les résultats et qui se trouve à l'extérieur du traitement. C'est quand même fascinant. Finalement, si l'on demeure critique, si l'on a les yeux ouverts, si l'on réfléchit devant les résultats, on va finir par accepter qu'il y a autre chose que nous ne comprenons pas et que la réponse de l'être humain, comme le disait Gilles, n'est pas uniforme, mais plutôt individuelle, quoique socialement influencée. Il faut s'occuper de l'être humain, et ne pas lui donner seulement un médicament. Malheureusement, l'analyse d'hier n'en est pas arrivée là, mais je pense que la science va finir par nous y mener. Et on peut dire aussi, comme critique à ce que je viens de dire, que la science a toujours montré des résultats négatifs. Mais personne ne s'en est intéressé et un jour, il va falloir qu'on s'y intéresse.

L'autre chose qui nous amène à réfléchir d'un point de vue beaucoup plus humain est le fait que désormais, on a transformé beaucoup de situations en situations chroniques et en général, 
en maladies complexes, parce qu'il y a conjugaison de plusieurs problèmes. Or, on ne peut pas répondre au chronique comme on répond à l'aigu et je pense que si on réfléchit là-dessus, ça va nous amener à notre rapport humain. Dans l'aigu, pour une otite à l'urgence, tu donnes un antibiotique et tu dis : "Au revoir! " Erreur! Parce qu'après trois jours, si tu fais une enquête, il n'y a plus personne qui prend l'antibiotique que tu as prescrit pour sept jours. Ça veut dire que le rapport ne s'est pas fait, que le médecin n'a pas fait adhérer le patient au plan de traitement. Mais comme les otites, finalement, peuvent guérir toute seules, ce n'est pas très grave.

M. Soulière : Comment le travail interdisciplinaire est-il affecté par la restructuration des établissements et de la réorganisation des services?

F. Alvarez : Dans le service et particulièrement dans l'équipe de transplantation, on vient d'horizons différents et avec des professions qui peuvent apparaitre différentes. Toutefois, on a un objectif commun et c'est le bien-être de l'enfant qu'on prend en charge. Chacun va apporter son savoir et son expertise. Dans l'équipe de transplantation hépatique, c'est intéressant de voir que dans la prise de décision de "transplanter " ou non un enfant, il y a autant de valeur accordée à la travailleuse sociale, qu'au psychiatre, au chirurgien ou à l'hépatologue. C'est pourquoi je préfere qu'on utilise le mot " interdisciplinarité ». On a d'autres groupes multidisciplinaires, on s'assoit, chacun donne son avis, selon sa spécialité et son point de vue, et le médecin prend la décision ultime. Cependant, ce mode de fonctionnement multidisciplinaire, ce n'est pas le mode de fonctionnement de notre équipe.

G. Bibeau : Vous savez, dans les hôpitaux, on a cassé les équipes. Si pour certaines situations cliniques, vous jumelez par exemple un pédiatre, un travailleur social, un psychologue et éventuellement d'autres professionnels, faut-il maintenir des équipes stables ou bien attribuer un case load à chacun des professionnels? Ça, c'est une décision très importante à prendre sur le plan organisationnel dans un hôpital. On a cassé les équipes, pas seulement ici à SainteJustine, mais partout au Québec. Personnellement, sur un plan anthropologique, je dirais que c'est grave de faire ça.J'ai toujours 
pensé que, compte tenu de la complexité des problématiques, avoir des équipes qui ont une certaine stabilité apporte beaucoup à tout le monde.

F.Alvarez: Je voulais aller dans ton sens en ajoutant que pour maintenir les équipes vivantes, il a fallu établir toute une méthodologie de résistance parce que dans cette société totalement individualiste et en même temps, dans une structure organisationnelle administrative basée sur une philosophie néolibérale qui se traduit en termes de rationalisation, rendement, etc., chacun doit donner son maximum. Pourquoi Alvarez, qui est le seul hépatologue pédiatre de tout le Québec, devrait-il passer du temps dans son équipe, à discuter des cas avec les membres de son équipe, alors qu'il doit poser des diagnostics, donner son avis sur plusieurs cas, etc.? Cette façon de faire la médecine n'a aucun bon sens! Ça veut dire qu'on te pousse à utiliser tes outils scientifiques et techniques plutôt que ta tête et tes sentiments. Et ça, pour moi, ça ne marche pas!

G. Bibeau: On a créé les spécialités en médecine et aujourd'hui on se rend compte, si on a un tout petit peu de vision, que dans vingt ans, le travail devra nécessairement se faire en équipe interdisciplinaire si on veut pouvoir prendre en charge les maladies complexes chez les patients. Cela veut dire que la pratique de la médecine va changer, même si on continue à appliquer les mêmes principes de la biomédecine. Il va toujours y avoir des gens qui vont analyser la situation et se rendre compte qu'avec les connaissances scientifiques et les principes de la biomédecine, on n'arrive pas à soigner en totalité. Quelquefois, on peut guérir, mais on n'arrive pas à soigner.

F. Alvarez: Et c'est ça l'élément clé de notre travail. La plupart des gens, ce qu'ils ont appris, tel que l'enseignent tous les manuels de médecine, c'est l'étiologie de telle maladie. Et comme disait Michel Serres: "On guérit un tout petit peu plus, mais pas tellement plus.» On n'arrive pas à soigner parce qu'on n'est pas préparé pour cela. Et ça, c'est fondamental. On doit soigner, et la guérison viendra. La réponse technique qu'on donne au problème médical comme seule réponse s'applique de moins en moins. Bien sûr, si un enfant avale une pièce de $1 \$$, on prend l'endoscope et une pince et on 
récupère la pièce : «Merci! Tu peux partir. " Mais encore là, on ne s'est pas intéressé à pourquoi l'enfant a avalé la pièce. On dit simplement : «Voilà, tu es guéri, tu pars! » Il faut en tenir compte. Parce que parfois, l'enfant revient dans nos services un an plus tard, dans la clinique sociojuridique, comme enfant maltraité.

M. Soulière: Et j'imagine que c'est à ce moment qu'interviennent les travailleuses sociales? Comment qualifieriez-vous leurs interventions?

F. Alvarez: Les travailleuses sociales, je crois, sont en défaut de formation. Je ne sais pas pourquoi, mais dans nos discussions, la DPJ apparait souvent. Ce n'est pas que je n'aime pas la DPJ, mais je ne crois pas que pour un seul de mes patients j'aie dû consulter ou avoir recours à la DPJ. J'ai eu parfois besoin d'une aide du CLSC. Il y a beaucoup de travailleuses sociales formées dans ce moule où les solutions sont surtout administratives. Pas toutes, évidemment. C'est très variable. L'avantage, c'est que par l'intégration des travailleuses sociales dans l'équipe, je vois des changements d'attitudes et d'approches. Elles sont contaminées par nous!

J'ai eu une expérience très intéressante avec une famille sénégalaise. Un enfant devait avoir une greffe intestinale à cause d'une maladie héréditaire. Les parents sont cousins germains. Et il s'agit de leur premier et seul garçon. Il y a aussi une fille plus grande et une fille plus jeune. Lui, il est dans le milieu et il est malade. Malade depuis la naissance. Le père est un universitaire, la mère est éducatrice dans une garderie. Vous imaginez que le dialogue avec ces parents est très facile. Ils parlent un excellent français, ils s'expriment très bien, ils sont très rationnels. Pourtant, jusqu'à très récemment, il n'y a eu que des conflits. Ils ont changé quatre fois de gastroentérologue et le dernier avait même appelé la DPJ, car il était désespéré. J'avais été invité à la réunion avec la DPJ. Et c'était extraordinaire, car la dame de la DPJ à un moment donné lui a dit: «Monsieur, il faut comprendre que nous, on est ici pour vous aider.» Le monsieur a dit : « Oui, madame. Maintenant, dites-moi comment vous allez nous aider. " Mais la dame de la DPJ ne savait pas quoi dire... Plus tard, les parents ont demandé de me voir et je les ai rencontrés à l'extérieur de cette réunion, dans mon bureau. Je leur ai dit : " Il me semble — corrigez-moi 
si je me trompe — que le problème était qu'il n'y avait personne qui vous a aidés à faire le deuil, d'abord le deuil de l'enfant idéal. Vous avez immigré, vous aviez des rêves et personne n'a écouté vos rêves. Ils ne savent pas pourquoi vous avez immigré, quelles sont les raisons. Et, votre enfant est né malade. Vous êtes cousins germains, c'est une maladie héréditaire et vous avez un sentiment de culpabilité que personne ne vous a permis d'évacuer. En plus, on vous fait sentir coupables des complications de votre enfant à cause de l'alimentation. " À partir de ce moment-là, il n'y a plus eu de conflits avec les parents. Personne n'avait essayé de construire une alliance thérapeutique avec eux. Ils agissaient comme technicien et avançaient qu'il y avait des différences culturelles. Or, il ne s'agissait pas ici de différences culturelles.

G. Bibeau : Est-ce que les choses ont changé? Et est-ce nous qui les avons fait changer? Je vais simplement proposer une courte réflexion à ce sujet. À l'époque de la Commission Rochon, j'ai dirigé une petite étude qui m'a amené dans les hôpitaux pour voir ce qui s'y passait. Je dois dire que les hôpitaux anglophones tels que le Jewish Hospital ou le Montreal Children Hospital étaient nettement en avance sur les hôpitaux francophones, qui étaient repliés sur eux-mêmes. La population immigrante allait surtout dans les hôpitaux anglophones : les Grecs, les Italiens, etc. Ils s'y sentaient mieux, davantage chez eux. Les hôpitaux francophones étaient nettement en retard, d'une façon incroyable. Et je l'ai écrit dans le rapport. Il fallait que les hôpitaux francophones et tout le réseau de la santé passent par une mutation importante. Or, les choses ont changé considérablement. Maintenant, dans la population consultante, dans la clientèle à l'hôpital, on a rattrapé à peu près ce qu'on trouve au Montreal Children Hospital.

Il y a vraiment des choses qui ont changé. Mais ce serait très présomptueux de notre part de penser que si l'hôpital SainteJustine a changé, c'est grâce à la petite intervention de l'UPI. Comme le démontrent les études sur l'efficacité : on ne peut jamais attribuer une amélioration ou un retour à la santé uniquement à l'intervention des médicaments. Ce n'est pas parce qu'un guérisseur a donné une potion à boire ou un médecin a prescrit 
un médicament que ce soit nécessairement cela qui explique l'amélioration ou la guérison de la personne malade. Ça peut être le sourire, la confiance donnée, l'appui qu'elle a reçu qui l'ont aidée à aller mieux. C'est un mouvement beaucoup plus puissant que l'UPI, beaucoup plus profond aussi, qui se passe dans l'ensemble de notre société, qui est en train de changer pas mal de manières de travailler dans les hôpitaux. Et l'UPI s'inscrit justement dans ce mouvement-là qu'il vient en quelque sorte dynamiser. Nous, à l'UPI, nous nous inscrivons dans cet effort collectif d'une société qui tente de devenir de plus en plus pluraliste. Et pour nous, cette pluralité, c'est dans l'espace de l'hôpital qu'elle se situe.

À mon retour d'Afrique en 1979,j'étais responsable à l'Université Laval d'un cours qui portait sur culture et santé et que j'ai donné aux étudiantes et étudiants en sciences infirmières, en ergothérapie et en médecine. Il y avait donc, déjà à cette époque, une sensibilité qui n'a cessé de grandir. Quand je regarde comment les programmes de formation des intervenants en santé se sont transformés - je pense aux sciences infirmières, notamment - en s'ouvrant de façon extraordinaire à autre chose qu'au biologique, c'est assez fascinant de voir ce qui est en train de se mettre en place. En service social à l’Université de Montréal, Gisèle Legault a été une des premières à contribuer grandement à ouvrir cette discipline. Mais il faut ajouter que ces deux professions ont aussi besoin de modèles, aussi bien en nursing qu'en service social.

F. Alvarez: Toutefois, si tu ne peux pas aller visiter les familles chez elles, tu peux les faire venir ici.Je vais t'expliquer une situation. Avant que je prenne la direction du programme de greffe, il y avait beaucoup de difficultés avec la population amérindienne, parce que les parents des enfants que nous voyions étaient alcooliques ou se droguaient. À un moment donné,je me suis demandé comment on pouvait changer cette situation et j'ai décidé de faire des réunions avec les familles. Ils viennent... Quand tu leur dis que c'est pour décider de la transplantation, ils viennent. Une fois, j'ai eu dans cette salle trois générations, et même des jeunes parmi lesquels un très jeune qui avait le même âge que mon patient. Je lui ai dit : "Toi, tu es qui? " Il a dit : «Moi, je suis le cousin. " J'ai 
dit : «Toi, tu vas t’assurer qu'il n'oublie jamais ses médicaments, même si vous sortez faire la fête.» À la fin,j'ai demandé qui allait assumer la responsabilité du suivi de cet enfant et nous garantir qu'il allait garder son foie. Il y avait toujours quelqu'un qui prenait la responsabilité. J'ai même fait un jour venir un chef de bande et je lui ai dit: "Vous êtes le chef de bande.Vous avez une responsabilité.» Et je l'ai amené dans la chambre d'un patient. Le père ne pouvait pas croire de voir apparaitre le chef de bande avec le médecin. Donc, quand tu ne peux pas aller à la maison, tu peux faire venir le monde. Et ils vont venir. Les gens se mobilisent et ils s'assument.

M. Soulière : Merci pour cette belle leçon de pratique! 